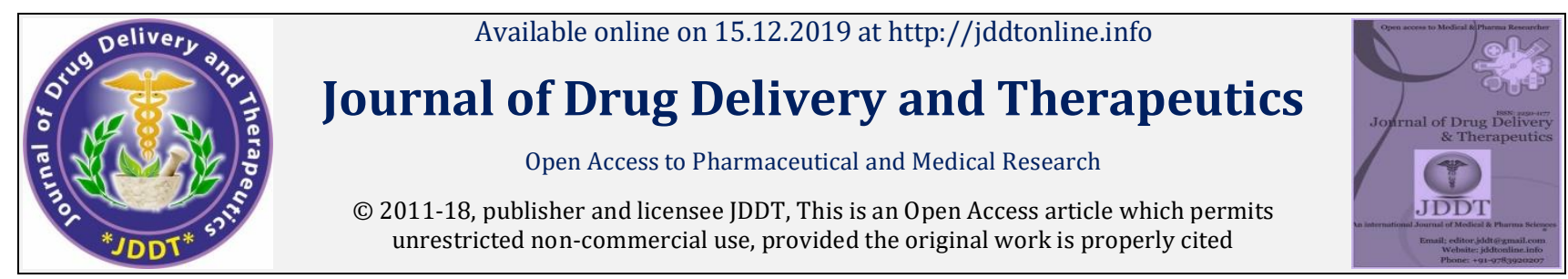

Open Access

Research Article

\title{
Optimization and Characterization of Fenugreek Seed Polymer Nanoparticles Loaded with Diltiazem HCl Nanoparticles by Desolvation Method
}

\author{
M. Gayatri Ramya ${ }^{*}$, Rajesh Akki ${ }^{2}$, Chakrala Jyothsna1 \\ 1. Department of Pharmaceutics, Acharya Nagarjuna University College of Pharmaceutical Sciences, Nagarjuna Nagar-522 510, Guntur, A.P, \\ India \\ 2. Hindu college of Pharmacy, Amaravathi road, Guntur-522002, Andhra Pradesh, India
}

\begin{abstract}
The main aim of the present investigation is to optimize and evaluate the fenugreek seed polymer nanoparticles using Diltiazem $\mathrm{HCl}$ as a model drug because Diltiazem $\mathrm{HCl}$ has short half-life. Nanoparticles were prepared by using desolvation method and evaluated to study the influence of polymer concentration and stirring speed on different characteristics of nanoparticles such as particle size, surface morphology, zeta potential, Encapsulation efficiency and In-vitro drug release. FTIR, DSC and XRD studies were also performed to determine the compatibility, degradation and crystalline nature of drug before and after formulation of nanoparticles. F7 (1:2 polymer concentration and 600 stirring speed) was optimized formulation based on its particle size $(672.1 \mathrm{~nm})$, encapsulation efficiency (83.1) having higher stability of Zeta potential value of -26.2, smooth surface morphology and having higher retarded drug release with non fickkian diffusion. By studying all the characteristics it was finally concluded that a natural polymer obtained from fenugreek seed can be used as a rate controlling polymer in the preparation of nanoparticles.
\end{abstract}

Keywords: Diltiazem $\mathrm{HCl}$, Fenugreek seed polymer, rate controlling polymer, Nanoparticles, Desolvation.

Article Info: Received 12 Oct 2019; Review Completed 23 Nov 2019; $\quad$ Accepted 29 Nov 2019; Available online 15 Dec 2019

Cite this article as:

Ramya MG, Akki R, Jyothsna C, Optimization and Characterization of Fenugreek Seed Polymer Nanoparticles Loaded with Diltiazem HCl Nanoparticles by Desolvation Method, Journal of Drug Delivery and Therapeutics. 2019; 9(6-s):155-163 http://dx.doi.org/10.22270/jddt.v9i6-s.3769

*Address for Correspondence:

M. Gayatri Ramya, Department of Pharmaceutics, Acharya Nagarjuna University College of Pharmaceutical Sciences, Nagarjuna Nagar-522 510, Guntur, A.P, India

\section{INTRODUCTION:}

In the recent trend all the researchers are making efforts to attain effective drug delivery. Polymeric nanoparticles are one of the emerging nanocarriers having the size range 10$1000 \mathrm{~nm}$.These polymeric nanoparticles were formulated using different polymers either from synthetic or from natural sources (Natarajan Jawahar et al., 2012; Konwar Ranjit et al., 2013). Based on the process employed for their formulation they can be classified into Nanospheres and Nanocapsules which are different in their structure and morphology. The drug may be either dispersed in the polymeric matrix or adsorbed on to the surface (Nanospheres) or encapsulated by a polymeric shell (Nanocapsules) (Ibrahim Khan et al., 2017).

Nowadays biodegradable polymers have vital role in delivering drugs due to their capability of circulating for an extended period of time to reach the target tissue which prevents toxicity to the remaining tissues (Aarti P.Nikam et al., 2014) Biodegradable polymers collected from natural sources such as plants and animals were utilized in formulation of several polysaccharides; fibers were used in formulation development. Pharmaceutical dosage forms. These natural polymers contain protein, mucilage. These polymers are mostly preferred over synthetic polymers due to their biocompatibility. They have significant role in delivering drugs, improve oral bioavailabilty, and prevents degradation of bioactive agents $(\mathrm{pH}$, enzymatic degradation). Mainly the mucilages which swell after coming into contact with fluid are used as several matrix formers, used in pharmaceutical carriers formulation and also helpful in controlling the drug release(Krishna LNV et al., 2011; Sujitha B et al., 2012; Kumar Vikas et al., 2011). Among several mucilages we are here discussing about mucilage obtained from seeds of fenugreek (Trigonella foenumgraecum) which was used as suspending agent, mucoadhesive polymer, tablet binder, gelling agent and rate 
controlling polymer in several formulations (Moumita chowdhury et al., 2017).

Diltiazem Hcl was chosen as a model drug which comes under calcium channel blocker. DTZ $\mathrm{HCl}$ has been widely used to treat chronic diseases like Angina pectoris, hypertension ( $G$ Aswinikumaret al., 2010) Because of its vasodilatory activity, this agent has been shown to improve the microcirculation in some tumors, thereby potentially improving the delivery of Anti neoplastic agents to tumor cells. The daily dosage recommended for it is $360 \mathrm{mg} /$ day in 6-8 hours interval and half-life of drug is only 3-5 hrs (Mikkilineni Bhanu Prasad et al., 2013). Hence prolonged release of Diltiazem helps in reduction of dosage frequency, more patient compliance and reduced side effects.

In this present investigation Diltiazem $\mathrm{Hcl}$ nanoparticles were formulated using Fenugreek seed mucilage as rate controlling polymer. Nanoparticles were prepared by using desolvation method by varying polymer concentration and stirring time and evaluated the effect of these variables on different parameters.

\section{MATERIALS AND METHODS}

\section{Materials}

Diltiazem $\mathrm{HCl}$ was supplied by Yarrow Chem Pvt Ltd (Mumbai). Fenugreek seeds were procured from local market. Dialysis tube was purchased from Sigma Aldrich (Mumbai). All other solvents and chemicals were of analytical grade.

\section{Extraction of Fenugreek seed mucilage:}

High quality fenugreek seeds were procured from local market. To remove dirt and debris fenugreek seeds were washed with distilled water. Then seeds were weighed and transferred to a beaker and required were quantity of double distilled water was added and soaked overnight. Then the soaked seeds were heated at $50^{\circ} \mathrm{C}$ for $2 \mathrm{hrs}$. To remove the marc from the mucilage the solution was filtered using multilayer muslin cloth and the resulted solution was reduced to $3 / 4$ th of the solution. The mucilage was separated using 3 volumes of Acetone and dried. Then the mucilage was sieved through \#no.60 and stored in desiccators for further use (senthilAdimoolam et al., 2018; Arun Verma et al., 2013; J.X. Jiang et al., 2007).

\section{Preparation ofNanoparticlesbyDesolvation Method:}

Nanoparticles were prepared by modified nanoprecipitation (desolvation) according to the method developed by Fessi and his colleague's. Fenugreek seed mucilage was dissolved in water and sonicated for 1 minute to remove aggregates. Then Diltiazem $\mathrm{HCl}$ was added to polymer solution to form aqueous phase. Then the acetone was added intermittently at the rate of $1 \mathrm{ml} / 5 \mathrm{~min}$ as a desolvating agent to the aqueous phase under continuous magnetic stirring at room temperature. 2-3 drops of 25\% Glutaraldehyde solution was added as a cross -linking agent after 10 minutes of acetone addition. Nanoparticles were formed spontaneously and this nanosuspension was stirred overnight for complete cross linking and in the meanwhile, acetone was also removed. 9 formulations were formulated by selecting 2 parameters at 3 levels 1) different drug: polymer ratios (1:1, 1:1.5, 1:2), 2) stirring speed $(600,900,1200 \mathrm{rpm})$. The prepared nanoparticles suspension was centrifuged for $2 \mathrm{hrs}$ at $6000 \mathrm{rpm}$. The nanoparticles pellet was washed repeatedly with acetone and resuspended in distilled water for further use (G Archana et al., 2015; RichinandanMaiti et al., 2018; Abhijeet P Pandey et al., 2017; Kai cheng et al., 2018).

\section{Characterization of Nanoparticles}

\section{Particle size analysis:}

Dynamic lightweight scattering technique (HORIBA, LB-550, Japan) was used to assess the mean particle diameter of the nanoparticles. The activity varied from $1 \mathrm{~nm}$ to $6 \mathrm{um}$ and the lightweight supply was $650 \mathrm{~nm}$ optical maser diode of $5 \mathrm{~mW}$. The samples of concerning 2.0 metric capacity unit liquid mixture dispersions were measured directly without pretreatment. Variety weighted means diameter was used to measure the particle size (A. Krishna sailaja et al., 2015).

\section{Zeta potential measurement:}

The Zeta potential (Surface Charge) which indicates the stability of the NP's can be defined as electrokinetic potential that is determined by electrophoretic mobility. Samples were prepared by diluting with water and corresponding zeta pontential were measured using Horiba Zeta Sizer $(A$. Krishna sailaja et al., 2015).

\section{Entrapment efficiency:}

The entrapment efficiency was measured while preparing the nanoparticles itself. The amount of Diltiazem $\mathrm{HCl}$ entrapped within nanoparticles was determined by measuring the amount of non-entrapped drug in supernatant recovered after centrifugation at 6000rpm for 30mins. Diltiazem $\mathrm{HCl}$ was measured spectrophotometrically at $\lambda \max 237 \mathrm{~nm}$. \% entrapment efficiency was calculated by using following formula (Ahmed Mohammed Nasef et al., 2017):

\section{Entrapment Efficiency Formula}

$\%$ entrapment efficiency

$$
=\frac{\text { amount of drug entrapped }}{\text { total amount of drug added }} \times 100
$$

\section{Differential Scanning Calorimetry (DSC) Analysis}

A DSC (Hitachi High-Tech Sciences-Model DSC7020, USA) (Andhra University, Vizag) was used to study the thermal properties of the pure drug,fenugreek seed mucilage powder and prepared nanoparticles in order to find out the possible drug polymer compatibility, melting point and degradation of drug. 4-5 mg of powder was sealed in an empty pre weighed aluminum sample pan and scanned in the temperature range of $20-300^{\circ} \mathrm{C}$ under an atmosphere of nitrogen rate of $10^{\circ} \mathrm{C} / \mathrm{min}$, followed by a cooling cycle back to rate of $20^{\circ} \mathrm{C}$ (GugulothuDhalapathi et al., 2018).

\section{X-Ray Powder Diffraction:}

X-ray diffraction (XRD) patterns of pure drug and nanoparticles were analyzed using the X-ray- diffractometer (Vignan University, Guntur). The powder samples, packed in rectangular aluminum cells, illuminated using $\mathrm{Cu}$ Ka radiation $(\mathrm{k}=1.54056 \AA)$ at $45 \mathrm{kV}$ and $30 \mathrm{~mA}$. Samples were scanned between diffraction angles of 6 to 800 . Scan steps of 0.008 were used and the $\mathrm{d}$ well time was $10 \mathrm{~s}$. A nickel filter was used to reduce the $\mathrm{Kb}$ contribution to the X-ray signal. The'd' spacing was computed according to Bragg's law of diffraction (GugulothuDhalapathi et al., 2018).

\section{Transmission Electron Microscopy (TEM):}

Transmission electron microscopy was used to study the surface morphology nanoparticles. Surface morphology of optimized formulation was done on a Philips EM268D instrument (Philips, Netherlands) at Ruska laboratories, Hyderabad. One drop of aqueous dispersed nanoparticles was placed on a carbon coated copper grid 400meshfollowed by negative staining with phosphotungstic 
acid solution ( $3 \% \mathrm{w} / \mathrm{v}$, adjusted to $\mathrm{pH} 4.7$ with $\mathrm{KOH}$ ) and placed at the accelerating voltage of $95 \mathrm{kV}$ for TEM(Nitan Bharti et al., 2014)

\section{In-vitro release studies:}

In order to perform the Invitro drug release studies of formulated nanoparticles. Suspension of nanoparticles was sealed in overnight soaked dialysis bag having molecular weight of $12000 \mathrm{Da}$ and then dipped in $100 \mathrm{ml}$ of $0.1 \mathrm{~N} \mathrm{HCl}$ as diffusion medium at $37 \pm 0.2{ }^{\circ} \mathrm{C}$. Samples $(5 \mathrm{ml})$ were removed at predetermined time intervals replaced with the same volume of fresh medium. The concentration of Diltiazem $\mathrm{HCl}$ was determined by UV spectrophotometer (Shimadzu UV-1601, Japan) at $237 \mathrm{~nm}$. The percent drug release was calculated using the calibration curve (Ahmed Mohammed Nasef et al., 2017).

\section{Release kinetics}

In order to understand the kinetics and the drug release mechanism, the results of in vitro drug release study of nanoparticles were fitted with various kinetic equations such as zero order (\% drug release vs. time), first order (log $\%$ drug unreleased vs time), Higuchi's model (\% drug release vs. square root of time) and korsemeyar peppas model to calculate mechanism of drug release (log\% drug release vs log time). $\mathrm{R}^{2}$ values were calculated for the linear curve for above plots.

\section{RESULTS AND DISCUSSION:}

\section{FT IR Spectral Analysis:}

The characteristic peaks were observed for pure drug physical mixture and nanoparticles. No new additional peaks were observed in physical mixture spectrum and nanoparticles FT IR spectrum. From the results it is clear that there was no drug and polymer interaction.
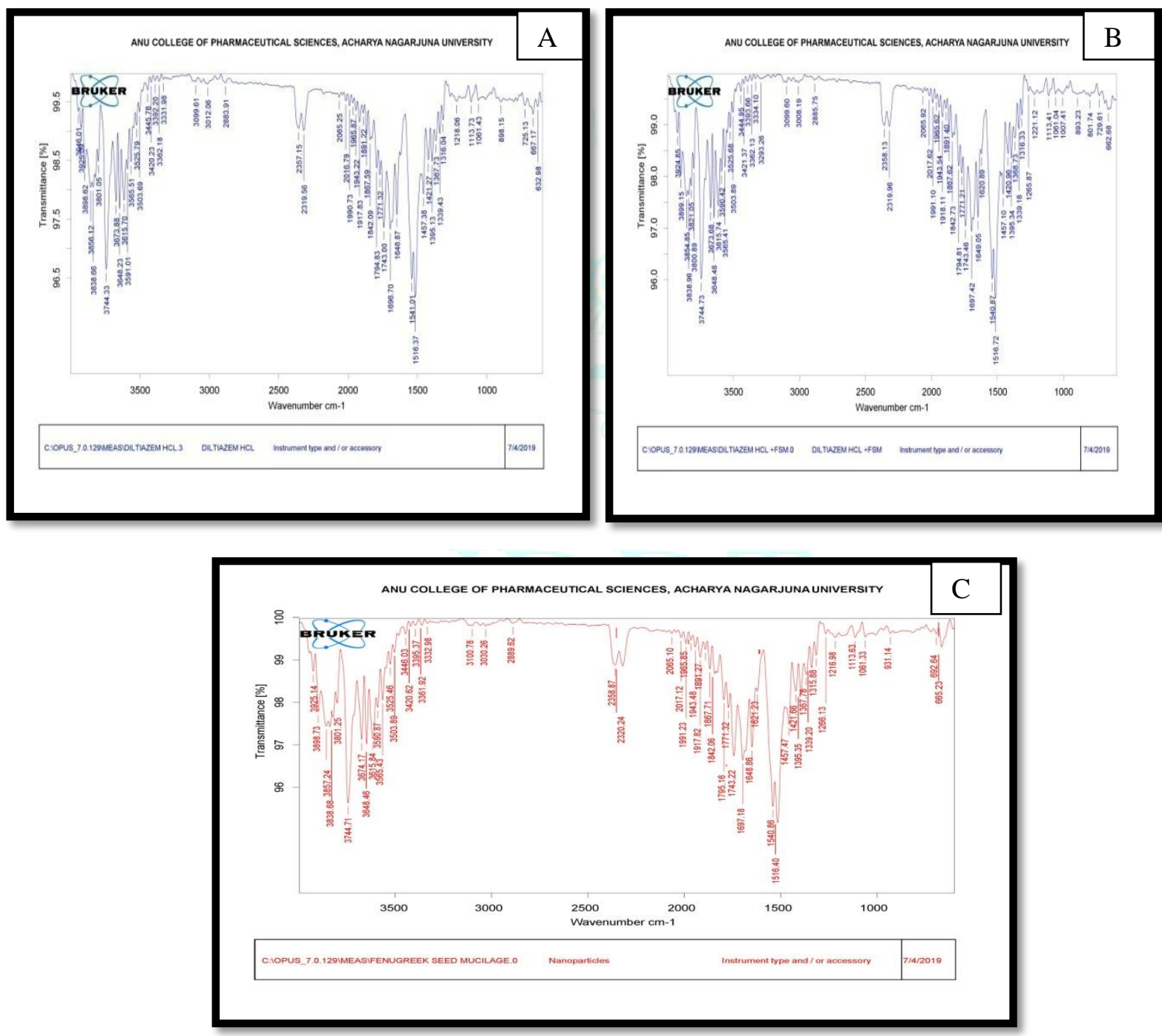

Figure 1: FT IR Spectral Analysis; A. Diltiazem HCl B. Physical mixture C. nanoparticles.

\section{Characterization of Nanoparticles}

\section{Particle size:}

Particle size of formulated nanoparticles was found submicron sized where the mean particle size of drug loaded formulations varied from 12.8 to $672 \mathrm{~nm}$.
Polymer concentration showed immense effect on mean particle size. As increase in polymer concentration the particle size of nanoparticles increases proportionally which could be due to increased viscosity that hampered diffusion of polymer from solvent to antisolvent.

Size of nanoparticles was significantly influenced by stirring speed. Increase in stirring speed resulted smaller particles as 
shown in the table. This reduction in nanoparticle size was due to enhanced mass transfer and rate of diffusion leading to rapid nucleation and precipitation.

\section{Polydispersibility index:}

Polydispersibility index of nanoparticles indicates the size distribution of nanoparticles in the distribution which indicates the stability of nanoparticles. The values in the range of 0.1 to 0.3 indicates monodisperse and 0.3 to 0.5 indicates broad distribution and value above 0.5 indicates very broad distribution which leads to particle aggregation and indicates less stability. The PDI values of all 9 formulations were found to be in the range of 0.177 to 0.953 .

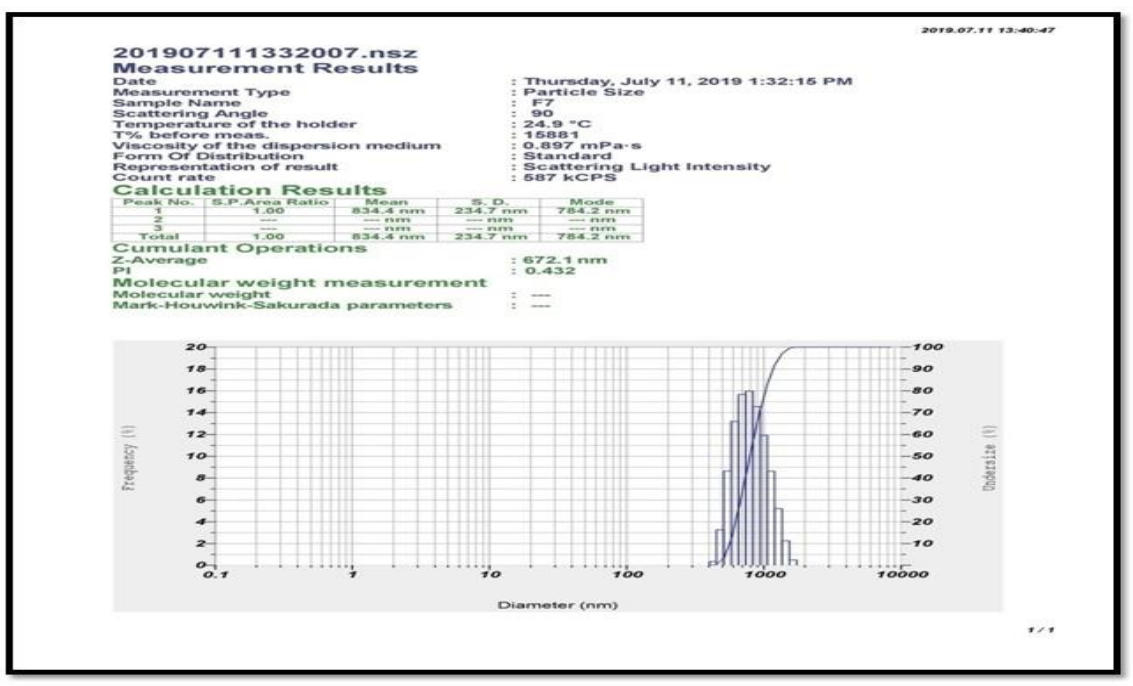

FIGURE 2: Particle size analysis of F7

\section{Zeta Potential:}

The zeta potential value measures the surface charge of nanoparticles which controls the stability of nanoparticles through strong electrostatic repulsion forces. The formulations obtained were of negatively charged because anionic nature of polymer. The normal range of zetapotential values were $<-30 \mathrm{mV}$ to $>30 \mathrm{mV}$. The zeta potential of the formulations was in the range of -0.7 to -26.2 $\mathrm{mV}$ which indicates higher stability of nanoparticles. The formulation F7 showed greater stability.

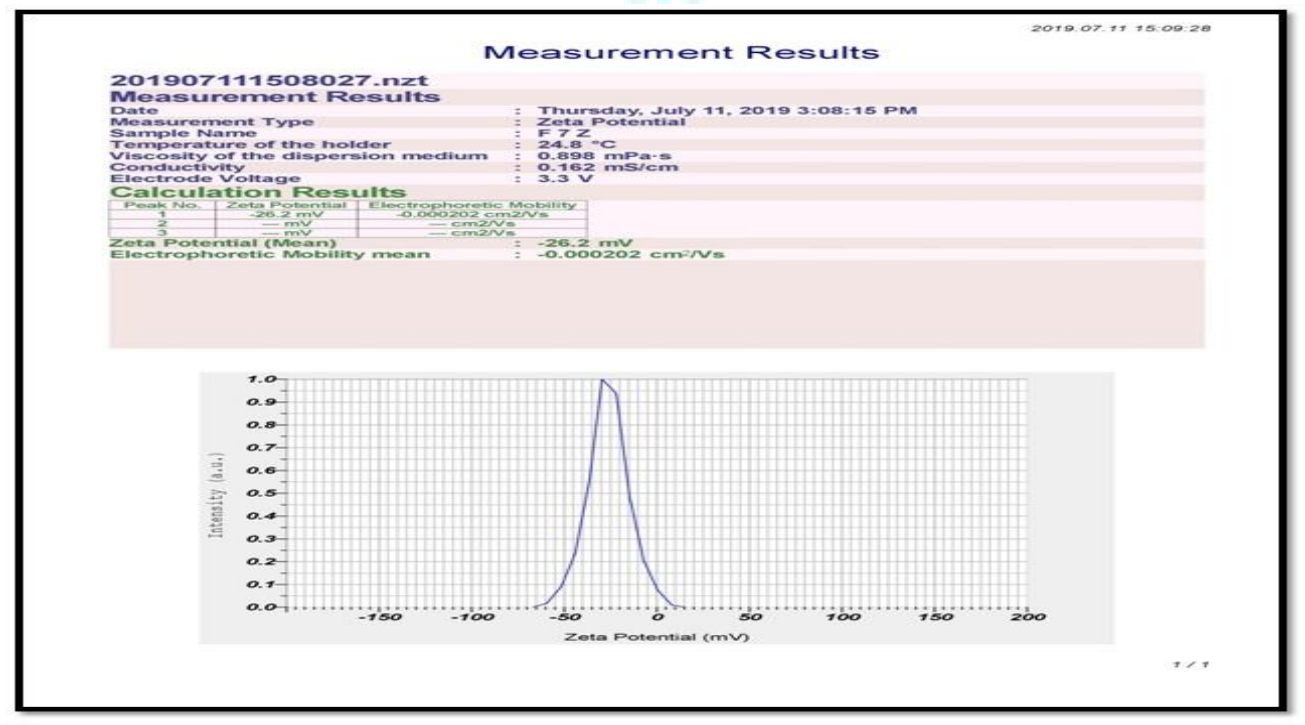

FIGURE 3: Zeta potential of F7 formulation

\section{Percentage entrapment efficiency and Loading efficiency:}

The percent entrapment efficiency of nanoparticles was in the range of 35.92 to $83.1 \%$ and the loading efficiency values of all nine formulations were found to be in the range of 19.7 to $38.6 \%$.

Both the loading and entrapment efficiency were significantly affected by polymer concentration used in the formulation. The \% entrapment efficiency and loading efficiencygradually increased due to increase in the polymer concentration. This is due to increase in viscosity of polymer solution which delays the diffusion of drug from the polymer layer which results in more entrapment. Stirring speed has negative effect on entrapment and loading efficiency of nanoparticles. As increase in stirring speed it results in lower particle size which results in higher surface area which makes the drug to leach out from nanoparticles 
TABLE 1: Characterization of nanoparticles

\begin{tabular}{|l|l|l|l|l|l|l|}
\hline S.NO & $\begin{array}{l}\text { Formulation } \\
\text { code }\end{array}$ & $\begin{array}{l}\text { Particle size } \\
\text { (nm) }\end{array}$ & $\begin{array}{l}\text { Polydispersibility } \\
\text { index }\end{array}$ & $\begin{array}{l}\text { Zeta potential } \\
\text { (mV) }\end{array}$ & $\begin{array}{l}\text { Entrapment } \\
\text { efficiency (\% E.E) }\end{array}$ & $\begin{array}{l}\text { Loading capacity } \\
\text { (\% L.C) }\end{array}$ \\
\hline 1 & F1 & 78.3 & 0.254 & -11.2 & 48.07 \\
\hline 2 & F2 & 56.7 & 0.349 & -2.2 & 41.26 \\
\hline 3 & F3 & 12.8 & 0.177 & -5.6 & 35.92 & 24.4 \\
\hline 4 & F4 & 297.9 & 0.953 & -1.7 & 58.37 & 19.7 \\
\hline 5 & F5 & 254.8 & 0.680 & -0.7 & 51.61 & 30.9 \\
\hline 6 & F6 & 0.788 & -13.3 & 48.75 & 28.5 \\
\hline 7 & F7 & 672.1 & -26.2 & & 83.1 \\
\hline 8 & F8 & 654.1 & 0.764 & -11.9 & 76.6 \\
\hline 9 & F9 & 563.6 & 0.516 & -16.8 & 64.9 \\
\hline
\end{tabular}

\section{DSC Studies:}

Thermal analysis was applied to study the physical state of the drug in the formulation and to check the drug-excipient compatibility. DSC was done for drug, polymer and optimized formulation. The sharp endothermic peak of Diltiazem $\mathrm{HCl}$ showed at $214^{\circ} \mathrm{C}$.

As there is no change in the peak of drug in formulation peak indicated that the polymer and drug shows no incompatibility.
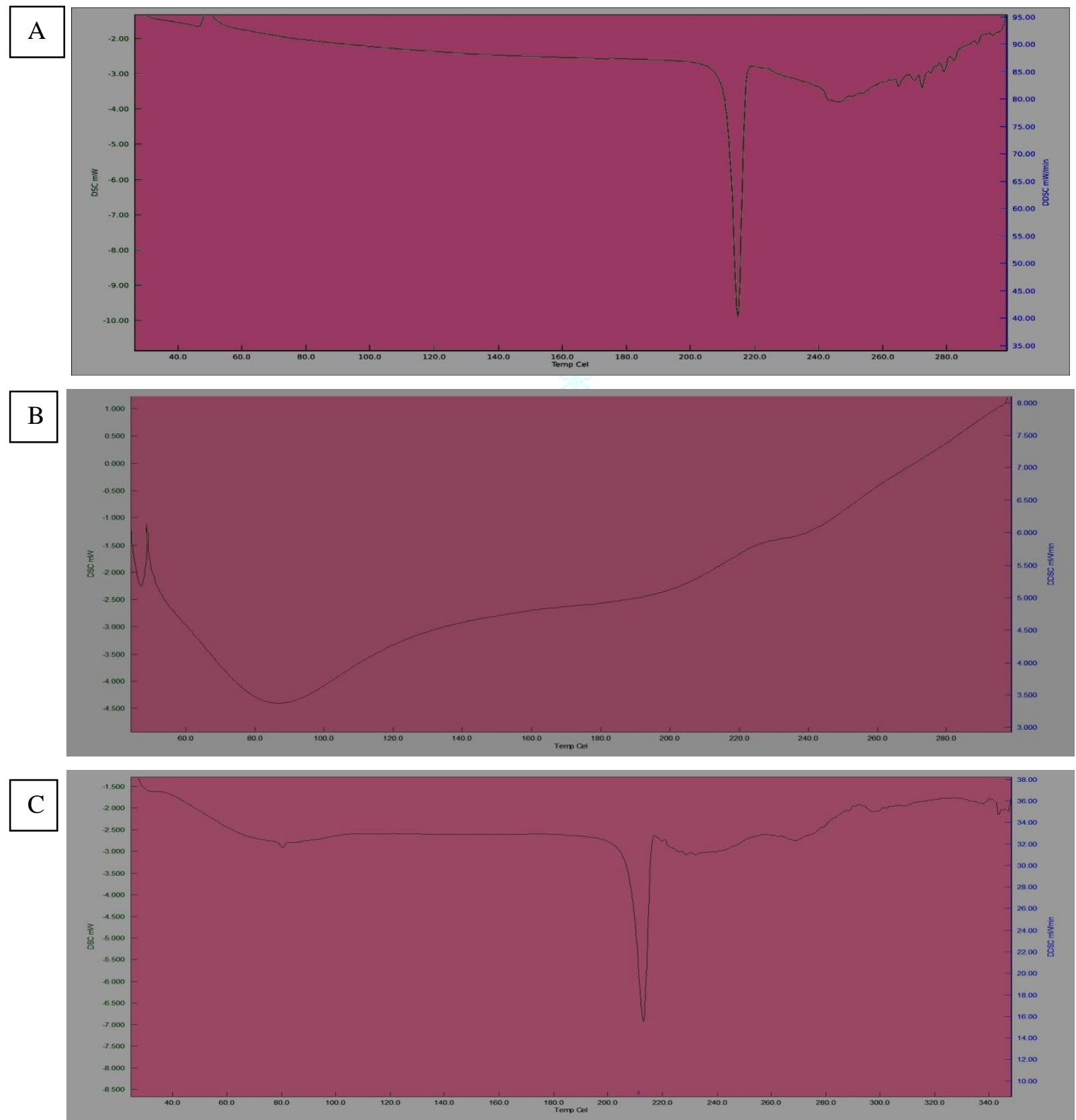

FIGURE 4: DSC studies A) Diltiazem $\mathrm{HCl}$ B) Polymer C) Nanoparticles 


\section{X - Ray Diffraction Studies:}

XRD studies were performed to study the influence of the polymers on phase transition of the drug in formulated nanoparticles. The XRD of Diltiazem $\mathrm{HCl}$ and optimized nanoparticles formulation F7 are shown in Fig.11. The result showed peaks Diltiazem HCl showed $2 \theta$ of 10.9791, 8.303, exhibiting similar peaks but with notably decreased intensity of the signal, showing no signs of chemical interaction between the drug and other formulation components.
Reduced intensity of the diffraction signal was probably caused by the dilution effect and decreased intensity of the drug was due to encapsulation into the polymer matrix. Diltiazem $\mathrm{HCl}$ displayed sharp peaks showing the crystalline phase while the drug containing microspheres showed less intense peaks indicating conversion of the drug crystalline phase to its amorphous phase.

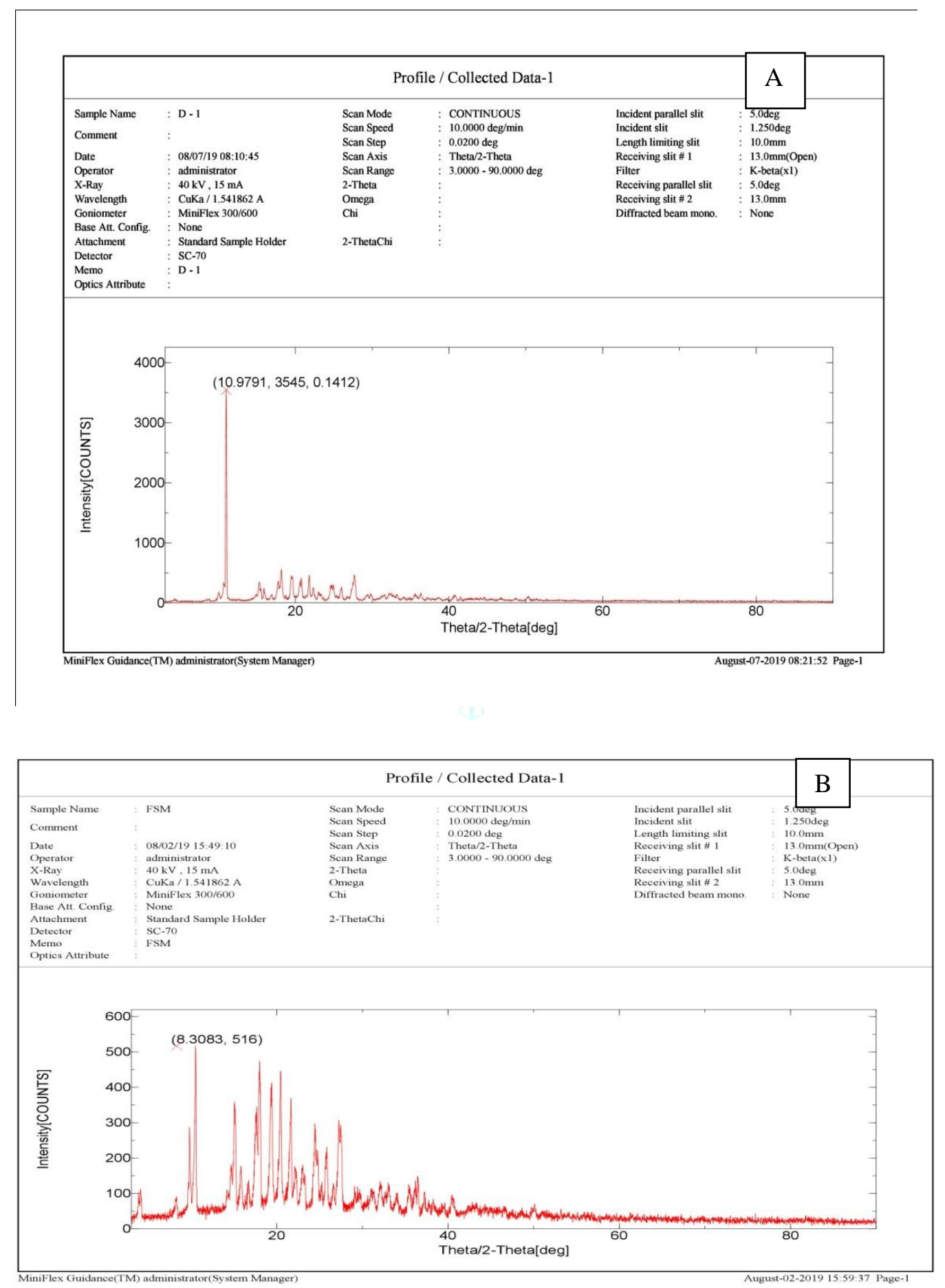

FIGURE 5: XRD studies of A) Drug B) Nanoparticles 


\section{TEM Analysis:}

TEM micrographs of prepared nanoparticle formulation showed that the nanoparticles were spherical in shape. The nanoparticles were found to be $593 \mathrm{~nm}$. The Transmission Electron Microscopy (TEM) confirmed the preparation of smooth and spherical nature of nanoparticles.
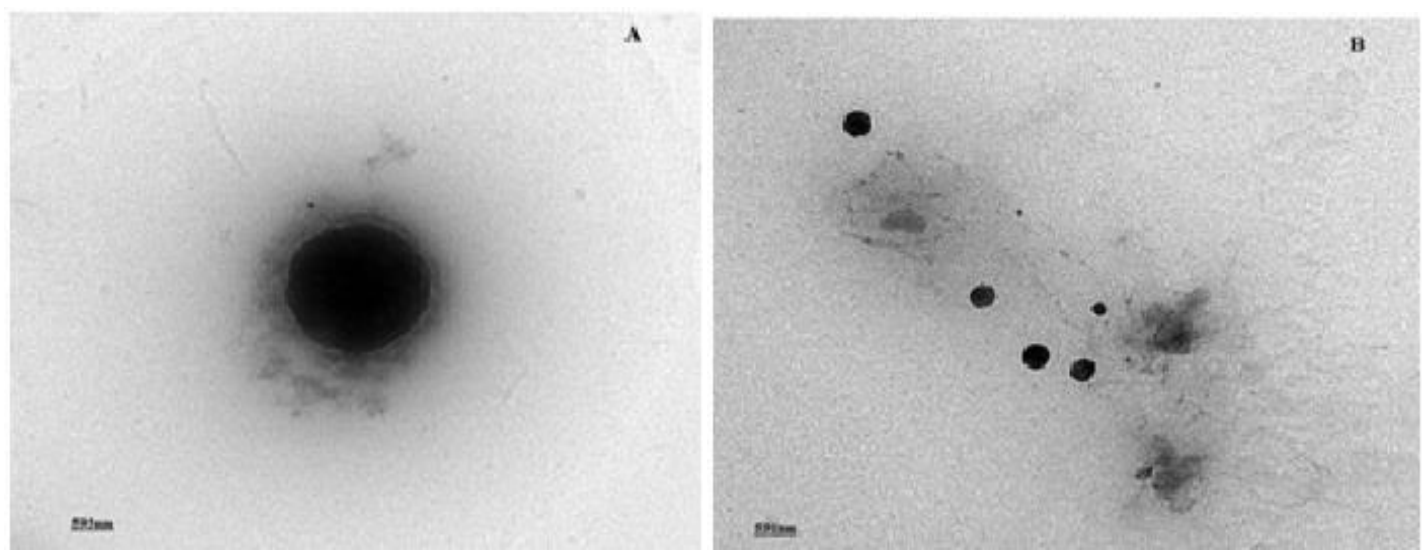

FIGURE 6: TEM micrographs of nanoparticles formulation

\section{In-vitro drug release studies:}

The effect of selected parameters on Invitro drug release studies of all formulations was evaluated. Initially all the formulations showed burst release of drug this may be due to the presence of drug near to surface of nanoparticles and then the drug release was gradually sustained.

Polymer concentration has immense effect on drug release from nanoparticles where, increasing polymer concentration resulted in decreased drug release rate. Formulation with drug: polymer ratio 1:2 showed lowest release rate. The percent of drug release decreased with increasing polymer concentration as shown in fig and this may be due to increase in polymer matrix at higher polymer concentration results in an increased diffusion path length.

Stirring speed has significant effect on drug release. From the study increase in stirring speed resulted in smaller particles through which drug release was faster from the nanoparticles and lower stirring speed resulted in larger particles which retarded the drug release from nanoparticles. This is because smaller particles have high surface area exposed to medium so drug release was faster from those nanoparticles.

\section{Release Kinetics:}

Formulations $\mathrm{F} 1$ to $\mathrm{F} 6$ showed greater $\mathrm{R}^{2}$ for Zero order so, the order of kinetics is zero order release and F7 to F9 showed greater $\mathrm{R}^{2}$ values for first order release so the order of kinetics is first order; this is due to increased polymer concentration which retarded the drug release. The Korsemeyars peppas release exponent (n) value lies in the range of 0.5 to 1 for all the formulations which showed the mechanism of release was anomalous non fickkian diffusion means diffusion along with swelling of polymer. The results were shown in the following table2:

TABLE 2: In vitro Release Kinetics

\begin{tabular}{|l|l|l|l|l|l|}
\hline $\begin{array}{l}\text { Formulation } \\
\text { code }\end{array}$ & $\begin{array}{l}\text { Zero order } \mathbf{R}^{2} \\
\text { values }\end{array}$ & $\begin{array}{l}\text { First order } \\
\mathbf{R}^{2} \text { values }\end{array}$ & $\begin{array}{l}\text { Higuchi } \\
\mathbf{R}^{2} \text { values }\end{array}$ & $\begin{array}{l}\text { Peppas release } \\
\text { exponent (n) }\end{array}$ & $\begin{array}{l}\text { Rate } \\
\text { constant }\end{array}$ \\
\hline F1 & 0.979 & 0.959 & 0.917 & 0.694 & 13.11 \\
\hline F2 & 0.957 & 0.926 & 0.933 & 0.640 & 13.20 \\
\hline F3 & 0.942 & 0.903 & 0.935 & 0.581 & 13.31 \\
\hline F4 & 0.982 & 0.938 & 0.871 & 0.691 & 10.15 \\
\hline F5 & 0.979 & 0.932 & 0.875 & 0.670 & 10.63 \\
\hline F6 & 0.973 & 0.931 & 0.859 & 0.641 & 10.97 \\
\hline F7 & 0.922 & 0.955 & 0.935 & 0.598 & 0.108 \\
\hline F8 & 0.931 & 0.978 & 0.951 & 0.618 & 0.124 \\
\hline F9 & 0.924 & 0.975 & 0.957 & 0.614 & 0.131 \\
\hline
\end{tabular}



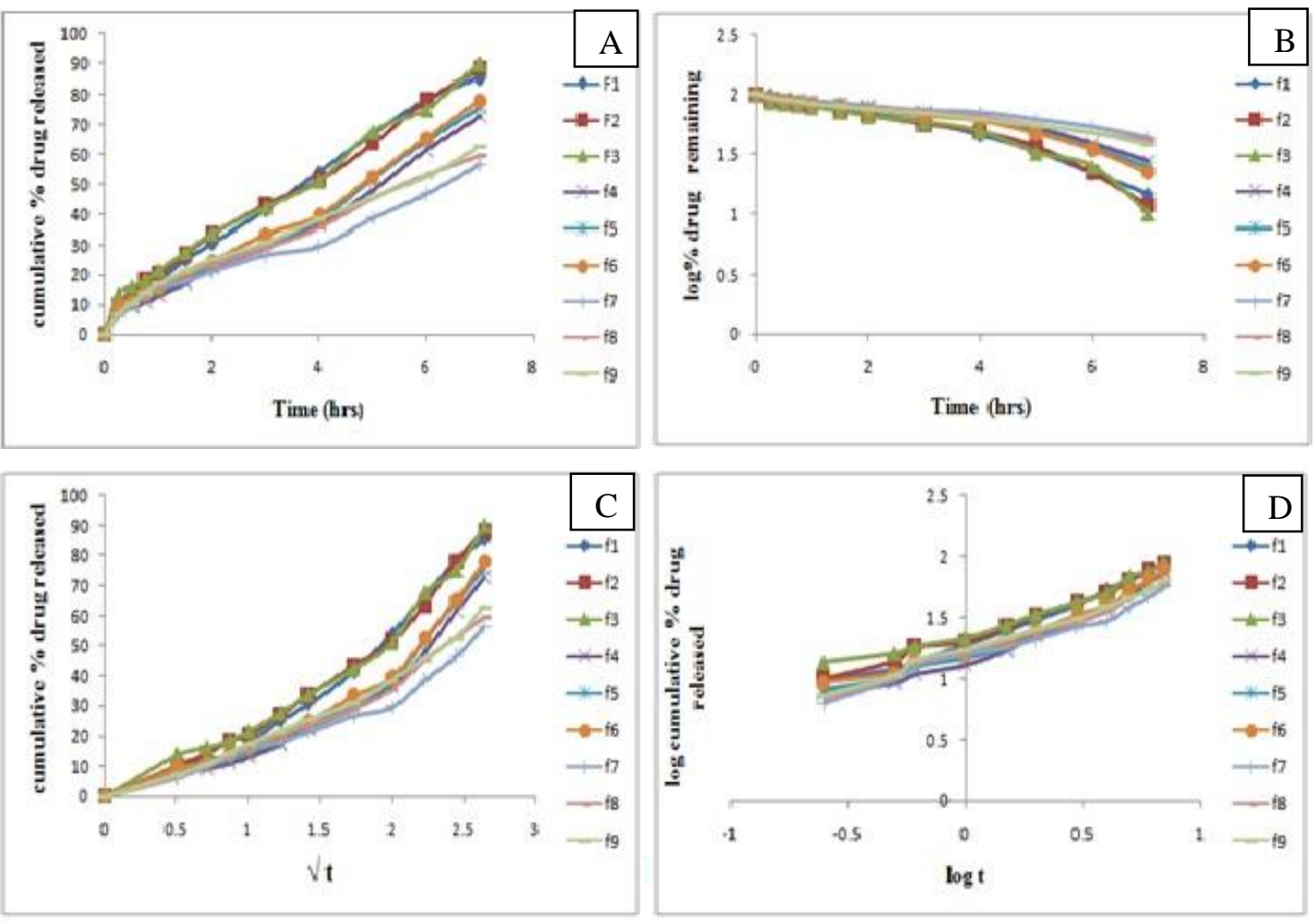

FIGURE 7: Invitro release studies A) Zero order release B) First order release C) Higuchi model D)Korsemeyars peppas

\section{CONCLUSION:}

The main use of the polymeric nanoparticles was to deliver the drug to the appropriate site and also to achieve the effective, controlled, protective drug delivery without any toxic side effects. By using this fenugreek seed polymer Diltiazem $\mathrm{HCl}$ which has short half-life was successfully incorporated to retard the drug release and prevent the drug degradation. The present research work revealed the use of fenugreek seed polymer as an effective rate controlling polymer in preparation of polymeric nanoparticles by desolvation method. From this study we concluded that the use of natural polymers for the preparation of nanoparticles is beneficial than toxic synthetic polymers.

\section{REFERENCES}

1. Natarajan Jawahar, Meyyanathan SN et al., Polymeric nanoparticles for drug delivery and targeting: A comprehensive review. Int. J. Health Allied Sci.2012; 1(4).

2. Konwar Ranjit, Ahmed Abdul Baquee et al., Nanoparticle: An Overview Of Preparation Characterisation and application. Int. Res. J. Pharm. 2013; 4(4): 47-57.

3. Ibrahim Khan, Khalid Saeed, Idress Khan et al., Nanoparticles: Properties, applications and toxicities. Arab. J. Chem. 2017.

4. Aarti P. Nikam, Mukesh.P. Ratnaparkhiand, Shilpa P. Chaudhari et al., Nanoparticles - An Overview. Int. J. Res. Dev. Pharm.L. Sci. 2014; 3(5): 1121-27.

5. Krishna LNV, Kulkarni PK, Dixit M, Lavanya D, Ravi PK et al., Brief introduction of natural gums, mucilages and their applicationsss innovel drug delivery systems. Int .J.Drug Formul Res 2011; 2(6): 52- 63.

6. Sujitha B, Krishnamoorthy B, Muthukumaran M. A role of natural polymers used in formulation of pharmaceutical dosage form. Int. J.Pharm Technol. 2012; 4(4): 2347-62.

7. Kumar Vikas, Sharma Arvind et al., Recent Advances in NDDS (Novel Drug Delivery System) For Delivery Of AntiHypertensive Drugs. Int. J. drug dev. res. 2011; 3(1).

8. P.B. Suruse, U.D. Shivhare, V.B. Mathur, K.L. Meshram et al., Development of Microcapsules of Glimepiride Using Fenugreek Seed Extract. Int. J. Pharm. Phytopharm. Res. 2013; 3(3): 212-15
9. Amit Kumar Nayak, Dillipkumar pal et al.,Trigonellafoenumgraecum L. seed mucilage-gellan mucoadhesive beads for controlled release of metformin $\mathrm{HCl}$. Carbohydra Polymers.2014; 107: 31-40.

10. Hemantakumar Sharma, Sunita Lahkar, Lila Kanta Nath et al., Formulation and in vitro evaluation of metformin hydrochloride loaded microspheres prepared with polysaccharide extracted from natural sources.Acta Pharm. 63; 2013: 209-222.

11. G Aswinikumar, Amit Bhat, Shoba Rani et al., Preparation and characterization Of Diltiazem Nanocapsules. Asian. J. Pharma. 2010; 224-34.

12. Senthil Adimoolam et al., Trigonella-foenum graecum l. seed mucilage-based mucoadhesive microspheres of diclofenac sodium.J.Anal. Pharm. res. 2018 ;7(2):114-119.

13. ArunVerma, Nitin Kumar, Rishabha Malviya, Subhamverma et al., Extraction and Evaluation of Colocasia esculenta andTrigonellafoenumgraecum L. Mucilage as a Pharmaceutical Adjuvant. African. J. Basic \&Appl.Sci. 2013;5 (6): 250-04.

14. J. X. Jiang, L.W. Zhu, W.M. Zhang, R.C. Sun et al., Characterization of Galactomannan Gum from Fenugreek (Trigonellafoenum-graecum) Seeds and Its Rheological Properties. Int. J. Polymer. mater. 2007; 56: 1145-54.

15. G.Archana et al.,Formulation and Evaluation of Naproxen Sodium Loaded Bovin SerumAlbumin Nanoparticles by Desolvation Technique. Asian J. pharm. Technol.innov. 2015; 3(14).

16. RichinandanMaiti, Saptarshipanigrahi, Yin Tingjie et al., Bovine Serum Albumin Nanoparticles constructing procedures on Anticancer Activities. Int. J.Adv. Res. Biol. Sci. 2018; 5(4): 226-39.

17. Abhijeet P. Pandey, Mahesh P. More, Kiran P. Karande, Ramesh V. Chitalkar et al., Optimization of desolvation process for fabrication of Lactoferrin snanoparticles using quality by design approach. Artif. Cells, Nanomed, Biotechnol. 2017; 45(6): 1101-14

18. Kai Cheng, Shaoping Sun, Xianfeng Gong et al., Preparation, Characterisation and anti-proliferative activities of biotin- 
decorated docetaxel-loaded bovine serum albumin nanoparticles. Braz. J. Pharm. Sci. 2018; 54(2): 1-11.

19. Krishna Sailaja A, P. Swati et al., Preparation of sodium alginate nanoparticles by desolvation technique using iso propyl alcohol as desolvating agent.Int. J. adv. Pharm. 2015; 4 (5): 60-71.

20. NitanBharti, S L Harikumar, Shishu, AbhisheikBuddiraja et al., Formulation and Evaluation OfGelatin Nanoparticles For Pulmonary Drug Delivery.World. J. Pharmacy. Pharm. sci. 2014; 3(12): 733-44.
21. Ahmed Mohammed Nasef, Ahmed RifaatGardouch, MomdouhMoustafaGhorab et al., Formulation and invitro evaluation of Pantoprazole loaded $\mathrm{PH}$ sensitive polymeric nanoparticles. Future. J. Pharm. Sci. 2017; xxx: 1-15.

22. GugulothuDalapathi, Choudhary Suraj kumar et al., Exploring ZiziphusSps fruit mucilages as a pharmaceutical excipient in Novel Drug Delivery Systems.J. Innov. Pharm.Sci. 2018; 2(2): 17-21. 